\title{
Desarrollo y validación de un método para la cuantificación de atorvastatina en tabletas mediante HPLC-DAD
}

Joseline Iveth Funes, Meidy Nairoby Meza ${ }^{1}$

Henry Daniel Ponce ${ }^{2}$

\section{RESUMEN}

Se propone un método para la identificación y cuantificación de atorvastatina (ATO) en comprimidos mediante cromatografía líquida de alta resolución con detector de arreglo de diodos (HPLC-DAD). Se optimizó la mejor separación del analito con una separación en gradiente isocratico, con una fase móvil compuesta de agua ajustando a pH 3.0 con ácido orto fosfórico y acetonitrilo (50:50).

El analito se logró separar utilizando una columna C18 de 150 × 4 mm, con un tamaño de partícula de $5 \mu \mathrm{m}$, a una temperatura de $40^{\circ} \mathrm{C}$ y la longitud de onda del detector fue de $245 \mathrm{~nm}$. El método fue validado siguiendo las recomendaciones recogidas por la Conferencia Internacional de Armonización (ICH), observándose buenas relaciones lineales para el analitos ( $r$ mayor que 0.998).

La precisión y exactitud de los resultados intra e interdías fue del 98.0 al $102 \%$. El procedimiento propuesto se aplicó con éxito para la determinación del compuesto en preparados farmacéuticos de comprimidos con buena exactitud y precisión (sin interferencia de excipientes), con lo cual el método se puede aplicar a los análisis de rutina del referido compuesto.

Palabras claves: atorvastina, HPLC, validación, tabletas.

${ }^{1}$ Estudiantes de la Carrera de Química y Farmacia, Facultad de Química y Farmacia, UNAH: iveth_funes94@hotmail.com

${ }^{2}$ Asesor, Departamento de Control Químico, Facultad de Química y Farmacia, UNAH: henry. ponce@unah.edu.hn 


\section{ABSTRACT}

A method for the identification and determination of Atorvastatine (ATO) in tablets by high performance liquid chromatography with diode array detector (HPLC-DAD) is proposed. Better separation of the analyte was optimized with a gradient isocratic separation by using a mobile phase composed of water adjusted to $\mathrm{pH} 3.0$ with orto phosphoric acid and acetonitrile (50:50). The separation of the analyte was achieved using a C18 column $150 \times 4 \mathrm{~mm}$ with a particle size of 5microns at a temperature of $40^{\circ} \mathrm{C}$ and a $245 \mathrm{~nm}$ detector wavelength. The method was validated following the recommendations made by the International Conference of Harmonization $(\mathrm{ICH})$, showing good linear relationships for the analyte ( $r$ greater than 0.998). The precision and accuracy of the results intra and inter-day was between 98.0 and 102\%. The proposed method was applied successfully to the determination of the compound in pharmaceutical preparations of tablets with good accuracy and precision (without interference excipients), with which the method can be applied to routine analysis based compound.

Key words: atorvastine, HPLC, validation, tablets. 


\section{INTRODUCCIÓN}

Una de las mayores preocupaciones a nivel mundial para la Organización Mundial de la Salud (OMS) son las enfermedades no transmisibles, las que constituyen un problema de salud pública que generan la erogación de elevadas cantidades de recursos financieros como consecuencia de los elevados costos en la atención necesaria para su tratamiento. Para el año 2004, un porcentaje del 32.2 de la población mayor de 20 años del Distrito Central, presentaba elevados valores de colesterol total, lo que incrementa el riesgo de padecer de enfermedades como la hipertensión y diabetes (Iniciativa Centroamericana de Diabetes, 2009).

La atorvastatina pertenece al grupo de medicamentos denominados estatinas, las cuales inhiben la 3-hidroxi-metil glutaril coenzima A (HMG-Co A), que es el paso que limita la tasa de biosíntesis del colesterol (Mahley y Bersot, 2003). La atorvastatina se administra como sal cálcica, con una dosis que puede ser entre los 10 y $80 \mathrm{mg}$ al día, para reducir los niveles lipídicos en pacientes con hiperlipidemia. La estructura molecular de la atorvastatina presenta muchos grupos funcionales de diferentes polaridades, en su forma de sal cálcica es ligeramente soluble en agua y acetonitrilo, con una valor de pKa igual a 4.46 y correspondiente número CAS 134523-03-8 (Moffat, Osselton y Widdop, 2005). La figura 1 muestra la estructura molecular de la atorvastatina y el espectro de absorción ultravioleta.

Figura 1. Estructura molecular y espectro de absorción ultravioleta de la atorvastina

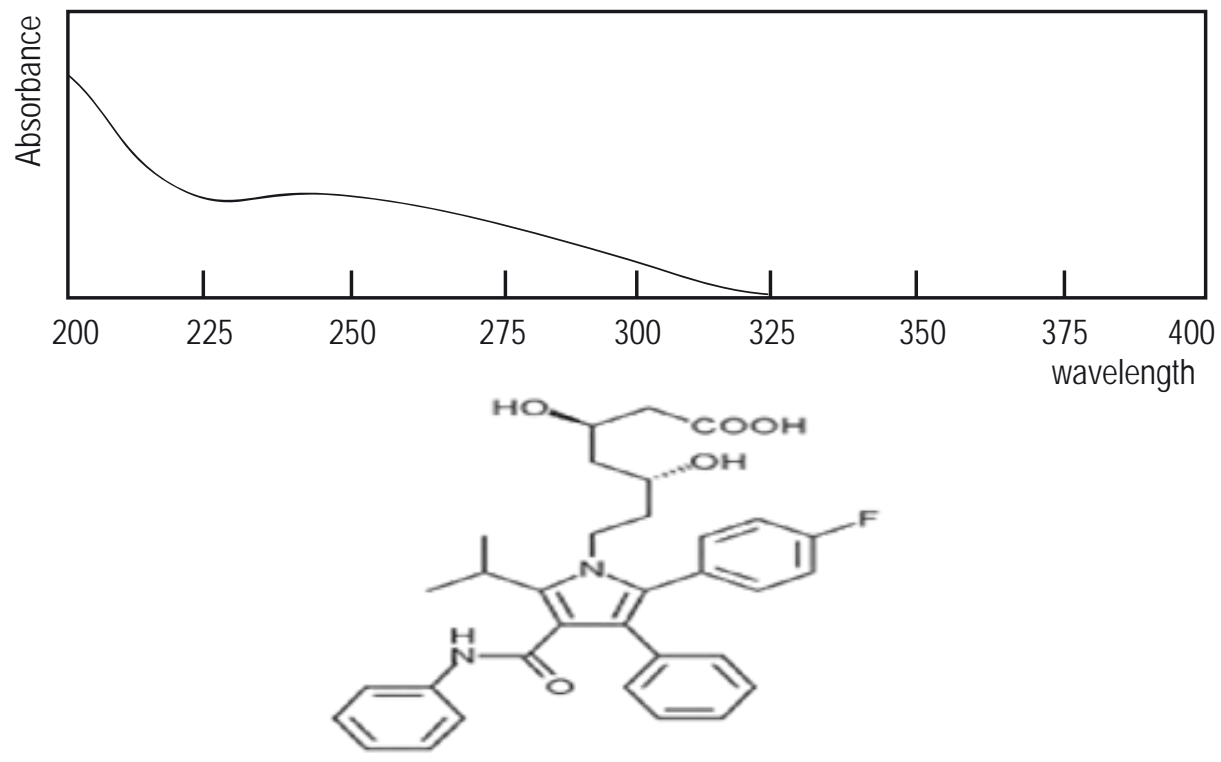


En nuestro país la gran parte de laboratorios farmacéuticos y de control de calidad de medicamentos utilizan como métodos de análisis los que contemplan los libros oficiales, entre ellos el más utilizado es el de la Farmacopea de los Estados Unidos (USP, por sus siglas en inglés), sin embargo, para el caso del análisis de atorvastatina en tabletas hasta el momento no se encuentra registrado ningún método de análisis. Es por ello que se hace necesario el desarrollo y posterior validación de un método analítico capaz de identificar y cuantificar la presencia de este compuestos en la referida forma farmacéutica.

En este contexto, la cromatografía líquida de alta resolución (HPLC) ha demostrado ser la metodología analítica más utilizada para el control de calidad de medicamentos, siendo aplicada con mayor regularidad en nuestro país, sin que esto signifique que sea un método barato y al alcance todos los laboratorios.

Algunos de los métodos que se han empleado para la determinación de atorvastatina en diversas matrices, han sido la cromatografía líquida acoplada a espectrometría de masas (LC-MS) y la cromatografía líquida con detector ultravioleta (Stanisz y Kania, 2006). La cromatografía es una técnica física de separación de compuestos presentes en una mezcla en la cual los componentes son distribuidos entre dos fases: una estacionaria y una móvil que se mueve en una dirección definida. En el caso de HPLC, la fase móvil es un líquido y la fase estacionaria puede tener características polares 0 apolares, pudiendo ser de diversos materiales, tales como silica, silica modificada, polímeros, entre otros.

La fase móvil actúa de portador de la muestra, la cual se presenta en solución que es inyectada en el instrumento y es arrastrada por la fase móvil. Los componentes de la solución emigran de acuerdo a las interacciones de los compuestos con las fases. Estas interacciones químicas determinan la separación de los constituyentes. La utilización de los diferentes detectores dependerá de la naturaleza de los compuestos a determinar y sus características químicas (Snyder, Kirkland y Dolan, 2010).

Uno de los aspectos más importante del desarrollo de métodos analíticos es la posterior validación de los mismos, proceso bajo el cual se demuestra, mediante experimentos y cálculos estadísticos, que el método funciona para el objeto que fue creado, en nuestro caso, para la identificación y cuantificación de atorvastatina en tabletas.

Para tal fin, existen diferentes recomendaciones de validación de métodos, entre las cuales las más importantes son las de la Comisión Internacional de Armonización (ICH), la cual establece los principales parámetros de desempeño a evaluar, lo mismo 
que los criterios que deben evaluarse (Guideline, 2006). A nivel regional, el Reglamento Técnico Centroamericano es el organismo que dicta las guías para la validación de métodos analíticos (RTCA, 2010).

Se desarrolló y validó un método por HPLC capaz de determinar la atorvastatina presente en tabletas y, finalmente, el método propuesto se utilizó para el análisis de un lote de tabletas comerciales.

\section{MÉTODOLOGÍA}

\section{Diseño}

El método de investigación se rige por un diseño aleatorio, ya que no se controlaron las cantidades de atorvastatina en las tabletas, sino que se trabajó con las tabletas comerciales adquiridas. También se realizó una revisión bibliográfica sobre el análisis de dicho compuesto en el ámbito farmacéutico, en donde se encontró que no existía ningún método oficial para su análisis en ninguna farmacopea o literatura oficial, sabiendo que la norma ISO-17025 establece como requerimientos para los laboratorios de ensayo y calibración la utilización de métodos validados.

\section{Población y muestra}

Lotes de tabletas de atorvastatina de $10 \mathrm{mg}$ y $40 \mathrm{mg}$ fueron proveídas por el laboratorio LANCASCO, con la marca COLVASTEN®. Se utilizó un estándar grado USP obtenido de los laboratorios docentes de la Facultad de Química y Farmacia, de la Universidad Nacional Autónoma de Honduras, para llevar a cabo todos los experimentos.

\section{Entorno}

El estudio se efectuó en la Facultad de Química y Farmacia en el laboratorio instrumental, en donde se encuentran el cromatógrafo líquido, baño ultrasónico, agitador mecánico, micropipetas y el resto del equipamiento de laboratorio.

Intervenciones

Para el desarrollo del método se utilizó un cromatógrafo líquido marca Shimadzu, modelo Prominence, que consiste en una bomba LC20-AT, un sistema de control 
CMB-20Alite, un inyector automático SIL-20A, un horno de columna CTO-A20 y un detector SPD-M20A. Se utilizó el software EZ Start para recolectar todos los datos obtenidos durante el proceso de investigación.

En el desarrollo de las condiciones cromatográficas se utilizó una columna Altima C18 de $150 \times 4.6 \mathrm{~mm}, 5 \mu \mathrm{m}$ de tamaño de partícula, a una temperatura del horno de $40^{\circ} \mathrm{C}$. El volumen de flujo de la fase móvil fue de $1 \mathrm{ml} / \mathrm{min}$ y el volumen de inyección se fijó en $25 \mu \mathrm{L}$ de las soluciones del estándar y la muestra. El detector de arreglo de diodos se controló a una longitud de onda de $245 \mathrm{~nm}$.

La fase móvil empleada consistió en una mezcla de acetonitrilo y agua acidificada con ácido orto fosfórico ajustando el pH a 3, en proporción final de 50:50. Todos los solventes utilizados fueron calidad HPLC de la marca J.T. Baker y se adquirieron en la casa comercial Labhospy, ubicada en Tegucigalpa.

\section{Análisis estadístico}

Para analizar todos los datos obtenidos de los experimentos del desarrollo y validación del método propuesto se utilizaron hojas de cálculo de Microsoft Excel 2010, además del software del instrumento, EZ Start versión 7.4; debido a que en el proceso de validación debe definirse qué será evaluado y cómo, es decir, cuáles criterios de calidad del método serán examinados y bajo qué criterios será realizada dicha evaluación. Todo ello dependerá de una serie de factores relacionados con las características de la matriz, complejidad del método analítico propuesto, exactitud y precisión con que deban expresarse los resultados y la aplicación que tendrá el método propuesto, entre otros aspectos. Para ello se siguieron las recomendaciones de la $\mathrm{ICH}$, las cuales aparecen en la tabla 1.

Tabla 1. Parámetros y criterios recomendados por la ICH

\section{Parámetro}

Especificidad

Linealidad

\section{Criterio}

a.Emplear un test de pureza de pico con un detector de arreglo de diodos (DAD)

a.Evaluación del gráfico de calibración lineal

b.Ecuación de la recta
c.Coeficientes de correlación $(r) \geq 0.998$ y determinación $(r 2) \geq 0.9998$

d.Análisis de varianza (prueba F) 


\section{Parámetro \\ Rango de trabajo a.Obtenido del ítem anterior, donde se cumplan los criterios de exactitud, precisión y linealidad \\ Exactitud (veracidad) a.Porcentaje de recuperación en el ensayo de la cantidad de analito reforzado, porcentaje de recuperación entre $90 \%-110 \%$ \\ Repetibilidad \\ a.Análisis de precisión en corto intervalo de tiempo: mismo día, instrumento y analista; evaluación de RSD (\%CV). El criterio será menor del $2 \%$ para el \%CV \\ Reproducibilidad intermedia \\ a.Análisis de precisión en condiciones amplias de variación: días, evaluación de RSD (\% CV); el criterio será menor $2 \%$ para el \%CV \\ b.Prueba t para la variación entre días}

\section{RESULTADOS Y DISCUSIONES}

\section{Desarrollo y optimización de la separación}

Uno de los aspectos más complejos e importantes al desarrollar un método mediante HPLC, es encontrar la fase móvil adecuada para lograr la separación del analito de interés, logrando una separación en menor tiempo, con menor uso de solvente orgánico posible y que el compuesto no co-eluya con interferentes e impurezas presentes en la muestra, es decir, buena selectividad. En esta etapa es necesario considerar la polaridad de la molécula, valor de pKa, así como grupos funcionales presentes, de modo que se encuentre el o los solventes con mayor afinidad y mejores resultados. En la presente investigación buscamos obtener un tiempo de análisis menor a los 15 minutos.

Las primeras pruebas fueron llevadas a cabo utilizando una columna C18 de $150 \mathrm{x}$ $4.6 \mathrm{~mm}$ y un tamaño de partícula de $5 \mu \mathrm{m}$, a temperatura ambiente. Se preparó una solución estándar de ATO con una concentración de $0.2 \mathrm{mg} / \mathrm{mL}$, inyectándose de la misma $100 \mu \mathrm{L}$ y colocando el detector a una longitud de onda de $245 \mathrm{~nm}$. Como fase móvil se empleó una mezcla de agua a pH neutro y metanol en proporción de 85:15 y a un volumen de flujo de $1 \mathrm{~mL} / \mathrm{min}$.

El cromatograma obtenido demostró que la ATO aparecía después de los 20 minutos, 
por lo que se cambió la proporción hasta un 30:70, con lo cual la cantidad de solvente orgánico era mucho mayor. En esas condiciones el pico del analito se mostró a los 7 minutos, sin embargo, se descartaron estas condiciones por el alto consumo de solvente orgánico.

Se decidió, entonces, modificar la concentración de prueba pasando a $0.1 \mathrm{mg} / \mathrm{mL}$, ya que con esta concentración la señal obtenida era suficientemente adecuada. Se utilizaron las mismas condiciones anteriores, solamente usando como fase móvil agua acidificada con ácido orto fosfórico y metanol, en la cual hasta una proporción de 40:60 se obtuvo el pico cromatográfico de la atorvastatina a los 10 minutos. En ese caso, siempre la cantidad de solvente fue elevada, aunque se demostró que el uso de agua acidificada mejoró sustancialmente la forma de los picos y el tiempo del análisis.

Finalmente, se realizaron pruebas preparando la fase móvil con agua acidificada y empleando como solvente orgánico acetonitrilo, el cual es un solvente con una mayor fuerza de elución y menor polaridad, lo que se espera logre una separación en menor tiempo y picos bien definidos. La proporción final a la que se obtuvieron las mejores condiciones de análisis fue empleando un $50 \%$ de cada solvente, apareciendo el pico de la atorvastatina antes de los 9 minutos, como se muestra en la figura 2.

Figura 2. Cromatograma obtenido con las condiciones finales del método

\begin{tabular}{|l||l|l|l|l|}
\hline $\begin{array}{l}\text { Retention Time } \\
\text { Area } \\
\text { Lambda Max } \\
\text { Width } \\
\end{array}$ & & & & \\
\end{tabular}


Finalmente, se optimizó el volumen de inyección, observándose una buena intensidad de la señal empleando $25 \mu \mathrm{L}$. Con estas pruebas se finalizó la etapa de optimización de la separación, quedando las condiciones finales del método mostradas en la tabla 2.

Tabla 2. Valores optimizados para la separación cromatográfica

\begin{tabular}{ll} 
Condición cromatográfica & Valores optimizados \\
\hline Columna & Alltima C18; $150 \times 4.6 \mathrm{~mm}, 5 \mu \mathrm{m}$ \\
Temperatura & $40{ }^{\circ} \mathrm{C}$ \\
Detector & $245 \mathrm{~nm}$ \\
Volumen de inyección & $25 \mu \mathrm{L}$ \\
Fase móvil & Agua acidificada con ácido orto fosfórico - \\
Volumen de flujo & $1 \mathrm{~mL} / \mathrm{min}$
\end{tabular}

Validación del método

Siguiendo las recomendaciones de la ICH se procedió a validar los distintos parámetros que aparecen en la tabla 1, excluyendo el límite de detección y límite de cuantificación, debido a que no son necesarios cuando el método que se utiliza lleva como finalidad la valoración del analito presente en las tabletas:

1. Especificidad: aprovechando que el detector con que cuenta el instrumento es uno de arreglo de diodos (DAD) y ya que se presentó la imposibilidad de contar con material de referencia de los productos de degradación y excipientes del preparado farmacéutico, se decidió evaluar la especificidad a través de la pureza de pico cromatografico, obteniendo para esto el espectro de absorción de la atorvastatina, con lo cual se pudo comprobar que el pico asignado al analito de interés corresponde adecuadamente a este y no hay ningún otro compuesto 0 interferente que co-eluya en el mismo tiempo de retención. En la figura 3 se presenta el espectro de absorción obtenido en el instrumento.

2. Linealidad y rango de trabajo: para la evaluación de la linealidad se preparó una curva de calibración por triplicado en días diferentes con concentraciones finales desde 10, 25, 50 y 100 partes por millón (ppm) y a partir de los datos obtenidos se construyó la recta de calibración, pendiente, intercepto, coeficiente de correlación (r) y de determinación (r2). La figura 4 muestra la recta de calibración en los tres días evaluados. 
Figura 3. Espectro de absorción obtenido para la pureza de pico cromatográfico

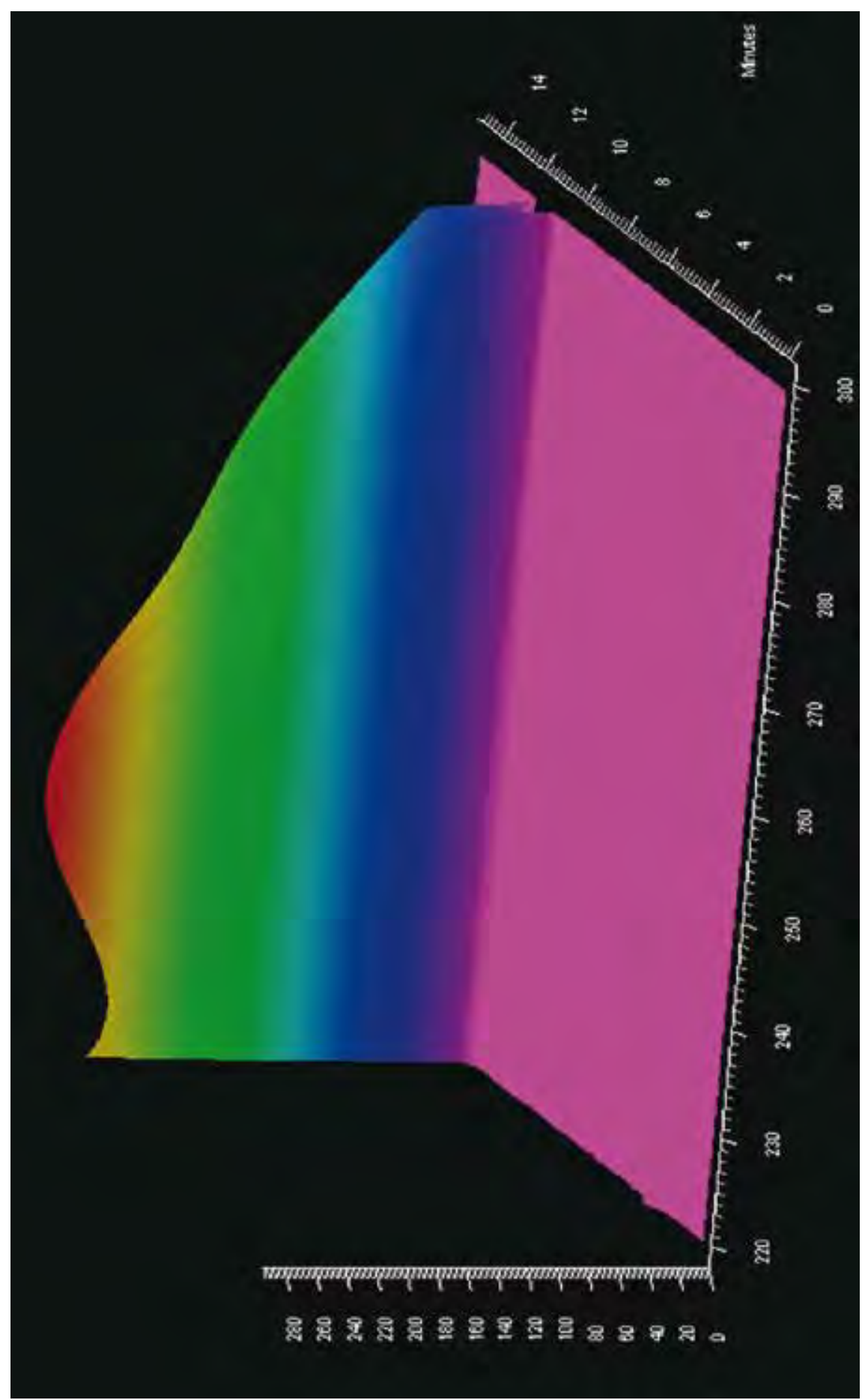


Figura 4. Curva de calibración para la evaluación de la linealidad y rango de trabajo

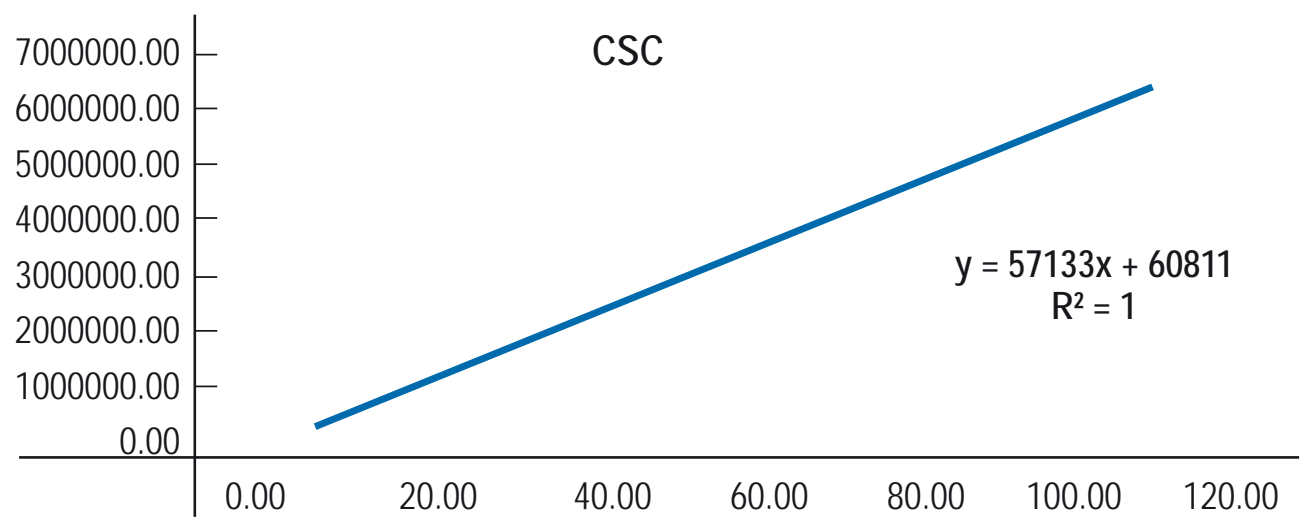

Al revisar los datos obtenidos de los gráficos se vieron valores de $r$ mayores a 0.998 y mayores a 0.9998 para el r2, adicionalmente la revisión visual demostró la correcta linealidad en el rango de trabajo seleccionado, así como el análisis de varianza (ANOVA). El valor de F calculado fue mayor al F tabulado, con lo cual se demostró que existe relación lineal entre las variables. La tabla 3 muestra los datos con los correspondientes criterios de evaluación para estos parámetros.

Tabla 3. Resultados encontrados para la linealidad del método

\begin{tabular}{|l|l|l|l|l|l|l|}
\hline Días & Pendiente & Intercepto & \multicolumn{1}{|c|}{ r } & \multicolumn{1}{|c|}{ 2 } & F calculado & F tabulado \\
\hline Día 1 & 60634.894 & 121759.10 & 0.9997 & 0.9995 & & \\
Día 2 & 50260.739 & 80040.36 & 0.9997 & 0.9994 & 202.2 & 0.339 \\
\hline Día 3 & 60593.155 & -19374.63 & 0.9999 & 0.9999 & & \\
Criterio & & & $\geq 0.998$ & $\geq 0.999$ & \multicolumn{2}{|c|}{ F calc > F tab } \\
Resultado & & & Cumple & Cumple & \multicolumn{2}{c|}{ Cumple } \\
\hline
\end{tabular}

3. Exactitud (veracidad): debido a las limitantes en cuanto a la cantidad de muestras y reactivos, se evaluó la exactitud fortificando seis muestras de tabletas que contenían atorvastatina, de la marca comercial Colvasten®, con concentraciones conocidas de una solución estándar del analito, para tener una concentración de fortificación de 49.88 ppm. Al evaluar los resultados, el porcentaje de recuperación promedio fue igual a $96.86 \%$.

4. Precisión: se evaluaron la repetibilidad y reproducibilidad intermedia del método, para lo cual se realizó el análisis de muestras de tabletas a concentraciones de 25, 50 y 100 ppm cada uno efectuada por triplicado, efectuadas por el mismo 
analista en el mismo día para la repetibilidad y en días diferentes para la reproducibilidad intermedia. Para el caso de la repetibilidad, todos los coeficientes de variación obtenidos fueron menores a 2 \%, como se muestra en la tabla 4.

Tabla 4. Resultados encontrados en la repetibilidad

\begin{tabular}{|cccc|}
$\begin{array}{c}\text { Concentración } \\
\text { (ppm) }\end{array}$ & $\begin{array}{c}\text { Porcentaje } \\
\text { promedio obtenido }\end{array}$ & $\begin{array}{c}\text { Desviación } \\
\text { estándar (S) }\end{array}$ & \% CV \\
\hline 25 & 95.62 & 0.6782 & 0.7093 \\
10 & 95.76 & 0.7184 & 0.7502 \\
100 & 98.41 & 0.4979 & 0.5060 \\
\hline Criterio & & & $<2 \%$ \\
\hline Resultado & & & Cumple \\
\hline
\end{tabular}

Finalmente, en la tabla 5 se muestran los resultados de las variaciones de los análisis efectuados entre días, incluyendo la prueba t para la reproducibilidad intermedia. En todos los casos se cumplieron los criterios de las pruebas estadísticas, siendo la t calculada en las tres concentraciones evaluadas menor que el valor tabulado, demostrando el método una buena reproducibilidad entre días.

Tabla 5. Resultados encontrados en la reproducibilidad intermedia

\begin{tabular}{|cccc|}
\hline \multicolumn{4}{c|}{ Datos día 1 } \\
\hline $\begin{array}{c}\text { Concentración } \\
\text { (ppm) }\end{array}$ & $\begin{array}{c}\text { Porcentaje } \\
\text { analito }\end{array}$ & $\begin{array}{c}\text { Desviación } \\
\text { estándar (S) }\end{array}$ & \% CV \\
\hline 25 & 95.620 & 0.678 & 0.427 \\
50 & 95.760 & 0.718 & 0.750 \\
100 & 98.410 & 0.498 & 0.506 \\
\hline \multicolumn{4}{c}{ Datos día 2 } \\
\hline $\begin{array}{c}\text { Concentración } \\
\text { (ppm) }\end{array}$ & Porcentaje & Desviación \\
\hline 25 & 97.690 & estándar (S) & \% CV \\
\hline 50 & 98.320 & 0.417 & 0.427 \\
100 & 98.150 & 0.987 & 1.004 \\
\hline
\end{tabular}




\begin{tabular}{|cccccccc|}
\hline \multicolumn{10}{c|}{ Datos día 1 } \\
\hline $\begin{array}{l}\text { Concentración } \\
\text { (ppm) }\end{array}$ & $\begin{array}{c}\text { Varianza } \\
\text { día 1 }\end{array}$ & $\begin{array}{c}\text { Varianza } \\
\text { día 2 }\end{array}$ & $\begin{array}{c}\text { n día } \\
\mathbf{1}\end{array}$ & $\begin{array}{c}\mathrm{n} \text { día } \\
\mathbf{2}\end{array}$ & $\begin{array}{c}\text { Varianza } \\
\text { agrupada }\end{array}$ & $\begin{array}{l}\mathrm{t} \\
\text { calculado }\end{array}$ & $\begin{array}{l}\mathrm{t} \\
\text { tabulado }\end{array}$ \\
\hline 25 & 0.460 & 0.174 & 3 & 3 & & -4.502 & \\
50 & 0.516 & 0.974 & 3 & 3 & 0.3169 & -5.568 & 0.2776 \\
100 & 0.248 & 1.254 & 3 & 3 & & 0.565 & \\
\hline
\end{tabular}

Aplicación para el análisis de tabletas

Para analizar tabletas de ATO de la marca comercial Colvasten® que reportan 40 miligramos, se empleó el método desarrollado, encontrándose $39.22 \mathrm{mg}$ por tableta para un porcentaje final de 98.05 de analito presente. La figura 5 muestra el cromatograma obtenido en el análisis de las tabletas.

\section{CONCLUSIONES}

Se logró desarrollar un método analítico aplicable al análisis de atorvastatina en tabletas, llevando a cabo la separación cromatográfica en gradiente isocrático, utilizando una fase móvil compuesta por agua acidificada con ácido orto fosfórico y acetonitrilo (50:50) con una columna C18, obteniendo la separación antes de los 10 minutos, empleando un detector de arreglo de diodos a $245 \mathrm{~nm}$.

Se validaron los principales parámetros siguiendo la guía de la $\mathrm{ICH}$, con lo que se demostró que el método analítico es simple, exacto, preciso y específico para el objeto que fue desarrollado, soportando los resultados mediante las pruebas estadísticas aplicadas, en donde se cumplieron todos los criterios establecidos. Se comprobó la aplicabilidad del método para el análisis de tabletas comerciales de atorvastatina, en donde los resultados cumplieron las especificaciones del fabricante y la normativa de la USP. 
Figura 5. Cromatograma obtenido durante el análisis de tabletas de Colvasten ${ }^{\circledR}$
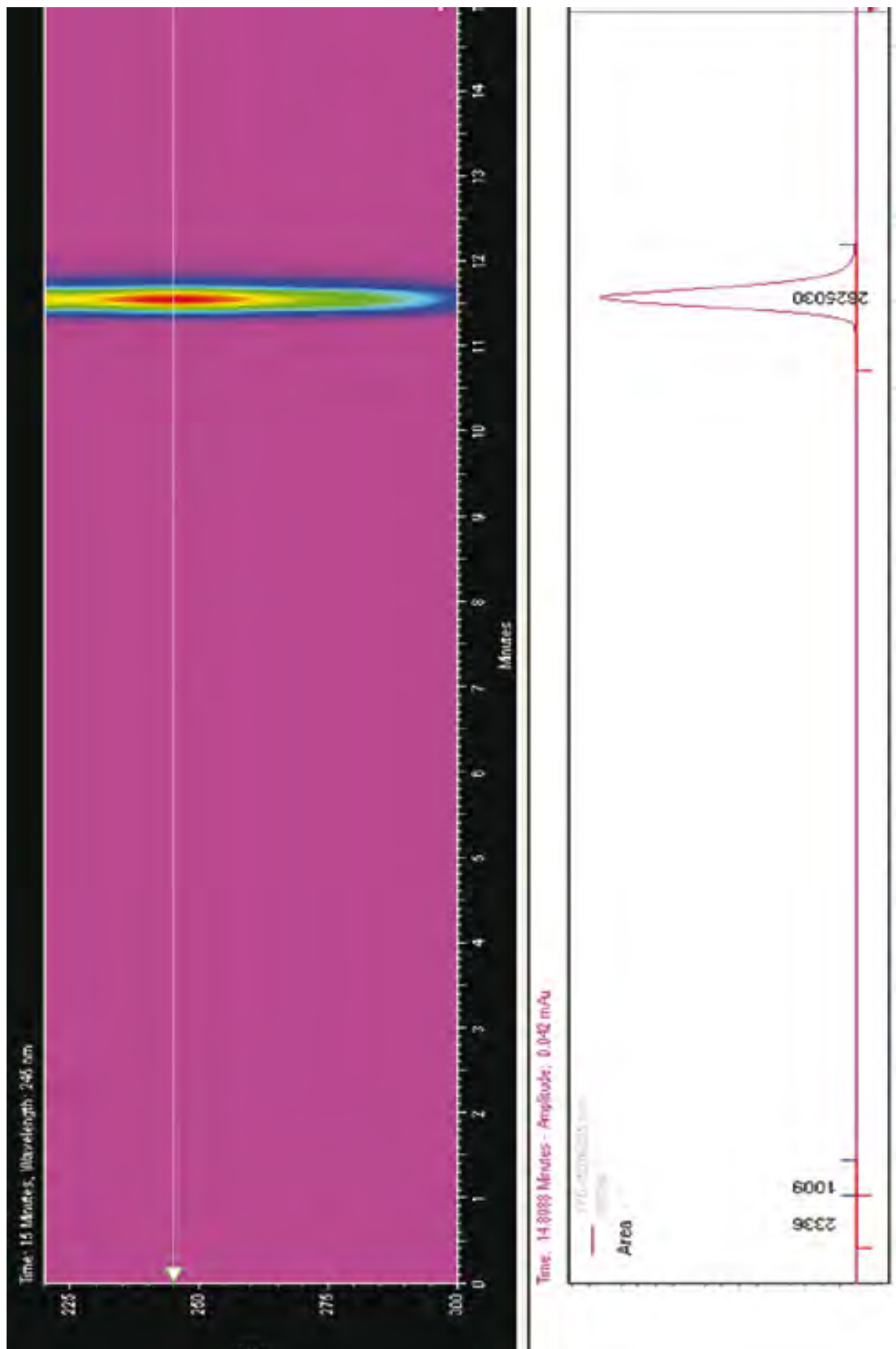


\section{AGRADECIMIENTOS}

A las autoridades de la Facultad de Química y Farmacia por la oportunidad de utilizar el cromatógrafo líquido del laboratorio instrumental, además de aportar todos los reactivos e insumos necesarios durante la experimentación. Asimismo, gracias al Grupo de Investigación Análisis Químico por compartir sus conocimientos y experiencias durante la investigación.

\section{BIBLIOGRAFÍA}

Guideline, H. T. (2006). Validation of Analytical Procedures: Text and Methodology. International Conference on Harmonisation of technical Requirements for Registration of Pharmaceuticals for human use. EE.UU.: ICH.

Iniciativa Centroamericana de Diabetes. (2009). Encuesta de diabetes, hipertensión y factores de riesgo de enfermedades crónicas. Tegucigalpa.

Mahley, R. W. y Bersot, T. P. (2003). Farmacoterapia para la hipercolesteremia y dislipemia. En Hardman, J.; Limbird, L. y Goodman Gilman, A. Las bases farmacológicas de la terapéutica. México: McGrawHill.

Moffat, A. C.; Osselton, M. D. and Widdop, B. (2005). Clarke's Analysis of Drugs and Poisons. Londres: Pharmaceutical Press.

RTCA. (2010). Validación de métodos analíticos para la evaluación de la calidad de los productos farmacéuticos. Reglamento Técnico Centroamericano. Tegucigalpa.

Snyder, L. R.; Kirkland, J. J. \& Dolan, J. W. (2010). Introduction to modern Liquid Chromatography. Estados Unidos de Norteamerica: Wiley Sons.

Stanisz, B. \& Kania, L. (2006). Validation of HPLC method for determination of Atorvastatin in tablets and for monitoring stability in solid phase. Acta Poloniae Pharmaceutica-Drug Research, 63(6), 471. 